\title{
Hyperhomocysteinemia is not associated with systemic arterial stiffness in patients with acute ischemic stroke
}

\author{
Kwang-Yeol Park, MD, PhD; Jeong-Min Kim, MD, PhD \\ Department of Neurology, Chung-Ang University Hospital, Chung-Ang University College of Medicine, Seoul, Korea
}

\begin{abstract}
Background:
Hyperhomocysteinemia is reported to be a risk factor for vascular calcification, atherosclerosis, and cerebrovascular disease. Also, arterial stiffness is associated with increased pulse pressure and cerebral small vessel disease. The aim of this study was to determine the association between hyperhomocysteinemia and brachial-ankle pulse wave velocity (baPWV) in patients with stroke.
\end{abstract}

\section{Methods:}

We identified consecutive patients with acute ischemic stroke who visited a tertiary university hospital within seven days of symptom onset between January 1, 2011 and April 30, 2017. The hyperhomocysteinemia was defined as serum level of homocysteine > $11 \mathrm{umol} / \mathrm{L}$. The average of baPWV measured on both sides was categorized into quartiles. The association between homocysteine and baPWV was tested using chi-square test and logistic regression analysis.

Results:

A total of 1624 patient were enrolled in this study. Mean age was 68.5 +/- 12.5 years and male was 882 (54.3\%). Hyperhomocysteinemia was found in $1074(66.1 \%)$. On bivariate analysis, baPWV was significantly associated with age, sex, hypertension, diabetes mellitus, smoking, systolic blood pressure, highly sensitive $\mathrm{C}$ reactive protein, estimated GFR, and hyperhomocysteinemia. However, the association between hyperhomocysteinemia and baPWV was not significant on multivariable logistic regression. The interaction term of hyperhomocysteinemia and sex was not significant, either. Age, hypertension, diabetes mellitus, and systolic blood pressure remained significant on multivariable analysis.

\section{Conclusion:}

Despite previous studies showed the association between homocysteine and arterial stiffness in general population, our study did not show significant association between them in stroke population. We presumed that potent vascular risk factors such as hypertension and diabetes rather than homocysteine play a more important role in damaging vessel wall in stroke patients. Further studies are warranted to confirm our findings and hypothesis.

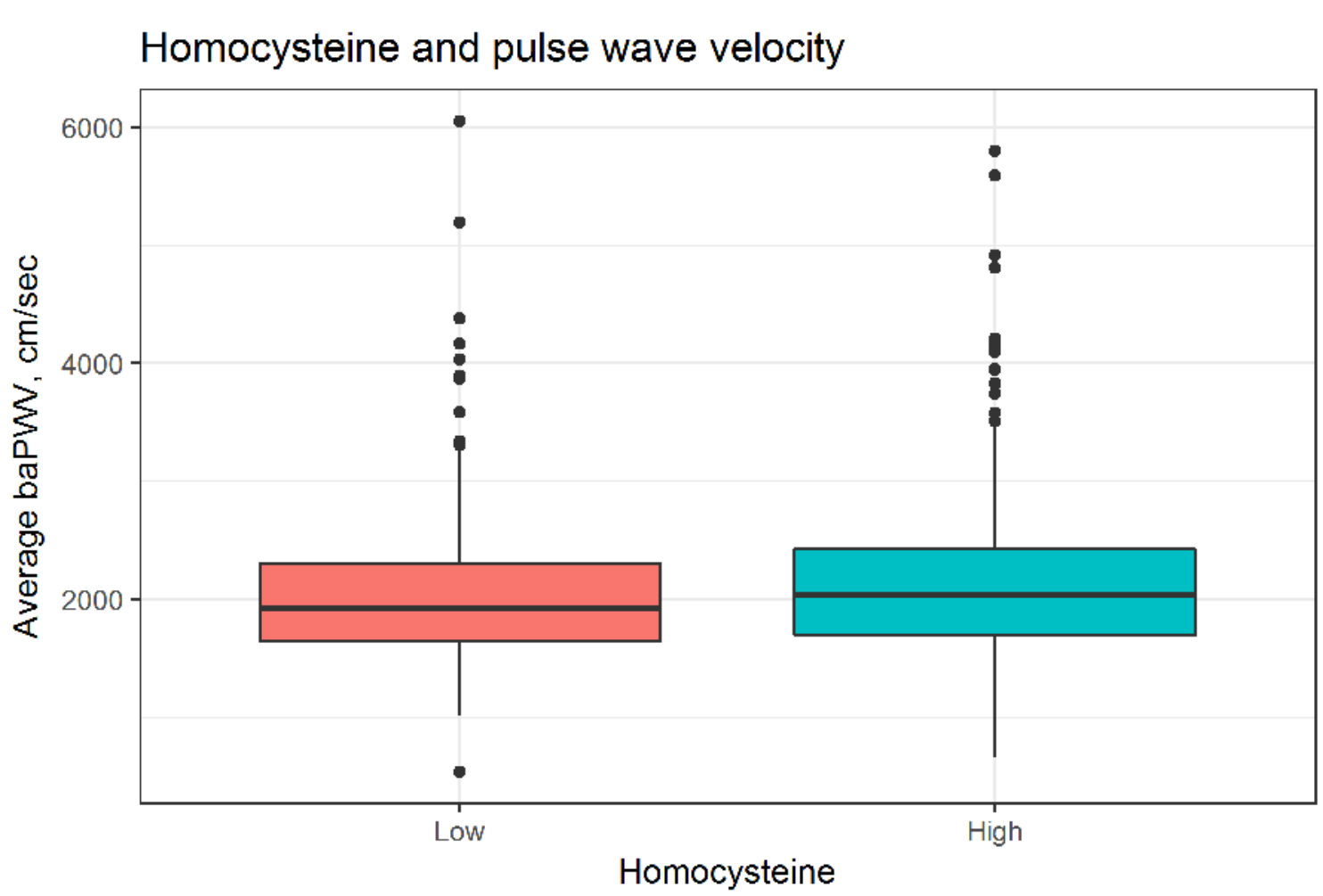

Table 1. Baseline information

\begin{tabular}{|c|c|c|c|c|c|c|}
\hline & Total & Q1 & Q2 & Q3 & Q4 & P_value \\
\hline Age & $68.46+/-12.46$ & $59.06+/-12.81$ & $67.09+/-10.96$ & $71.23+/-9.77$ & $76.48+1-8.92$ & 0.000 \\
\hline Sex & $882(54.3 \%)$ & $268(66 \%)$ & $249(61.2 \%)$ & $209(51.6 \%)$ & $156(38.4 \%)$ & 0.000 \\
\hline HTN & 969 (59.7\%) & $150(36.9 \%)$ & $247(60.7 \%)$ & $265(65.4 \%)$ & $307(75.6 \%)$ & 0.000 \\
\hline DM & $482(29.7 \%)$ & $65(16 \%)$ & $120(29.5 \%)$ & $138(34.1 \%)$ & $159(39.2 \%)$ & 0.000 \\
\hline $\mathrm{IHD}$ & $160(9.9 \%)$ & $37(9.1 \%)$ & $46(11.3 \%)$ & $43(10.6 \%)$ & $34(8.4 \%)$ & 0.479 \\
\hline Af & $341(21 \%)$ & $74(18.2 \%)$ & $78(19.2 \%)$ & $96(23.7 \%)$ & $93(22.9 \%)$ & 0.146 \\
\hline Smoking & $414(25.5 \%)$ & 150 (36.9\%) & 129 (31.7\%) & $95(23.5 \%)$ & $40(9.9 \%)$ & 0.000 \\
\hline HL & $214(13.2 \%)$ & $49(12.1 \%)$ & $50(12.3 \%)$ & $63(15.6 \%)$ & $52(12.8 \%)$ & 0.428 \\
\hline PrevCVA & $272(16.7 \%)$ & $53(13.1 \%)$ & $69(17 \%)$ & $75(18.5 \%)$ & $75(18.5 \%)$ & 0.124 \\
\hline NIHSS & $4.56+/-5.73$ & $4.3+1-5.89$ & $4.45+/-5.78$ & $4.41+/-5.43$ & $5.09+/-5.81$ & 0.203 \\
\hline SBP & $151.25+/-27.69$ & $142.54+/-25.39$ & $150.96+/-25.1$ & $153.43+/-27.03$ & $158.06+1-30.66$ & 0.000 \\
\hline DBP & $86.74+/-14.22$ & $84.87+/-13.95$ & $86.8+/-13.44$ & $86.87+/-14$ & $88.45+/-15.26$ & 0.005 \\
\hline hsCRP & $7.26+1-21.28$ & $5.15+/-19.19$ & $5.9+/-15.28$ & $7.36+1-20.62$ & $10.62+/-27.76$ & 0.001 \\
\hline FBS & $135.29+/-56$ & $126.58+/-54.12$ & $133.75+/-49.19$ & $139.68+1-58.35$ & $141.17+/-60.72$ & 0.001 \\
\hline HDA1C & $6.21+/-1.38$ & $5.92+/-1.25$ & $6.23+/-1.47$ & $6.37+/-1.44$ & $6.33+/-1.31$ & 0.000 \\
\hline BUN & $18.13+1-8.22$ & $16.44+1-6.03$ & $18.09+/-7.9$ & $18.57+/-8.4$ & $19.43+/-9.83$ & 0.000 \\
\hline $\mathrm{Cr}$ & $0.98+/-0.82$ & $0.9+1-0.6$ & $0.96+/-0.64$ & $1.06+/-1.17$ & $0.99+/-0.77$ & 0.043 \\
\hline eGFR & $87.38+/-27.34$ & $102.26+/-24.3$ & $88.7+/-26.78$ & $82.19+/-25.32$ & $76.34+/-25.97$ & 0.000 \\
\hline T_chol & $179.34+/-45.09$ & $180.3+/-43.13$ & $179.02+/-42.79$ & $178.19+/-45.61$ & $179.85+1-48.69$ & 0.915 \\
\hline HDL & $46.36+/-13.29$ & $46+/-11.29$ & $45.68+/-13.47$ & $46.78+/-12.95$ & $47+/-15.17$ & 0.446 \\
\hline LDL & $106.35+/-33.23$ & $107.27+/-31.96$ & $108.7+/-32.11$ & $104.39+/-33.71$ & $105.03+1-34.98$ & 0.223 \\
\hline TG & $117.54+/-77.05$ & $119.56+/-90.64$ & $116.36+/-68.74$ & $116.47+/-72.63$ & $117.76+/-74.61$ & 0.928 \\
\hline svo & 331 (22.9\%) & $74(22.2 \%)$ & $78(21.5 \%)$ & $92(24.9 \%)$ & $87(22.8 \%)$ & 0.718 \\
\hline Homocystein & $14.01+/-6.95$ & $13.62+/-7.11$ & $14.23+/-8.43$ & $14.31+/-6.48$ & $13.9+/-5.43$ & 0.467 \\
\hline Homocystein_High & $1074(66.1 \%)$ & 254 (62.6\%) & 255 (62.7\%) & 278 (68.6\%) & $287(70.7 \%)$ & 0.024 \\
\hline VitD & $14.84+/-8.48$ & $15.5+/-8.46$ & $14.79+/-7.6$ & $14.8+/-8.02$ & $14.26+/-9.69$ & 0.258 \\
\hline VitD_adj & $14.82+/-8.33$ & $15.52+/-8.3$ & $14.83+/-7.32$ & $14.7+/-8.01$ & $14.23+/-9.52$ & 0.207 \\
\hline
\end{tabular}

Table 2. Multivariable analysis

\begin{tabular}{lrrrrrr} 
& Estimate & Std. Error & t value & $\operatorname{Pr}(>|t|)$ & $2.5 \%$ & $97.5 \%$ \\
\hline (Intercept) & -102.8261377 & 144.1666924 & -0.7132448 & 0.4758074 & -385.6203473 & 179.968072 \\
\hline Age & 21.0968699 & 1.2266007 & 17.1994607 & 0.0000000 & 18.6907970 & 23.502943 \\
\hline SexM & -76.3997440 & 27.6731318 & -2.7607914 & 0.0058378 & -130.6827500 & -22.116738 \\
\hline eGFR & 0.2550936 & 0.5807549 & 0.4392449 & 0.6605486 & -0.8841024 & 1.394290 \\
\hline HTNHT(+) & 130.7906977 & 26.6190895 & 4.9134174 & 0.0000010 & 78.5752779 & 183.006117 \\
\hline DMDM(+) & 115.2098656 & 27.5637650 & 4.1797579 & 0.0000309 & 61.1413911 & 169.278340 \\
\hline SmokingSmoking(+) & -54.0549188 & 31.6355737 & -1.7086752 & 0.0877221 & -116.1105637 & 8.000726 \\
\hline SBP & 4.4923627 & 0.4410054 & 10.1866378 & 0.0000000 & 3.6272961 & 5.357429 \\
\hline hsCRP & 1.4951541 & 0.5834227 & 2.5627286 & 0.0104841 & 0.3507249 & 2.639583 \\
\hline VitD_adj_3gr1 & -71.3896158 & 28.6658476 & -2.4904066 & 0.0128693 & -127.6199113 & -15.159320 \\
\hline VitD_adj_3gr2 & -26.8295846 & 35.1092082 & -0.7641752 & 0.4448854 & -95.6990343 & 42.039865 \\
\hline Homocystein_High & 13.1124857 & 28.7318694 & 0.4563743 & 0.6481882 & -43.2473166 & 69.472288
\end{tabular}

